\title{
Investigation into an efficient numerical modelling approach for estimating path-loss over variable terrain
}

\author{
Nazabat Hussain *, Asif Iqbal, Varun Jeoti \\ Affiliati Department of Electrical \& Electronics Engineering \\ Universiti Teknologi PETRONAS Bandar Sri Iskandar 32610, Perak Malaysia \\ *Corresponding author E-mail:nazabat@hotmail.com:
}

\begin{abstract}
Different studies have been conducted for radio wave propagation in troposphere using different numerical modelling approaches. The most reliable approach is based on parabolic wave equation (PWE). The modelling of PWE is approached using different numerical schemes that include Split-step Fourier Transform Method (SSFM), Finite Element/Difference method and Wavelet based numerical method. The conventional Finite Element/Difference method are less accurate and/or computationally more expensive. While in comparison, split-step wavelet method (SSWM) is highly accurate and computationally very efficient. The SSWM has been previously used for modelling of PWE with smooth terrain. However, the real conditions are completely different as they contain variable terrain. The irregularities in surface of terrain have considerable influence because of reflection and diffraction on radio-wave propagation. In order to develop an effective communication system, a model that properly incorporates the reflection parameters from the variable terrain. In this research work, the SSWM is proposed for modelling of PWE for radio wave propagation over variable terrain. In SSWM, compactly supported wavelet of Daubechies 6 are used as bases. Obtained results accurately accounts the reflection from rough terrain surface and shows good agreement with SSFM.
\end{abstract}

Keywords: Communication, Split-step wavelets, numerical, wireless, evaporation duct.

\section{Introduction}

Band of frequencies in EM spectrum used for wireless communication starts from $9 \mathrm{KHz}$ to $275 \mathrm{GHz}$. The frequency of wave less than $9 \mathrm{KHz}$ are not used because of limited bandwidth and large antennas size. Similarly, the frequency of wave for more than $275 \mathrm{GHz}$ are not used because of very high attenuation [1], [2]. The propagation of radio wave in any medium is characterized in terms of parameters such as frequency, wavelength, electric field strength $(E)$, magnetic field strength $(H)$, electric polarization, velocity of propagation (v), refractive index and Poynting vector [3], [4]. In order to analyze the properties of an antenna, an isotropic antenna is required. Ideally, the radiation of radio waves with isotropic antenna propagates in all directions. Let the power radiated by an isotropic antenna indicates by $P_{t}$ and received power $P_{0}$ after covering a distance $\boldsymbol{d}$ can be obtained as [5],

$$
P_{0}=\frac{P_{t}}{4 \pi d^{2}}=\frac{E_{0}^{2}}{2 \eta_{0}}
$$

where $P_{t}$ is transmitted power in Watt, $E_{0}$ is magnitude of electric field in $\mathrm{V} / \mathrm{m}, \boldsymbol{d}$ is distance from the transmitter antenna in $m$ meter and $\eta_{0}=120 \pi \Omega$ is free space intrinsic impedance. The received power can be expressed as,

$$
P_{r}=\frac{P_{t} \times G_{t}}{4 \pi d^{2}} \times \frac{G_{r} \times \lambda^{2}}{4 \pi}=\frac{G_{r} \times P_{t} \times G_{t} \times \lambda^{2}}{(4 \pi d)^{2}}
$$

Where $G_{t}$ and $G_{r}$ are the transmitter and receiver antenna Gain respectively, $\lambda=c / f$ is wavelength and $c$ is the speed of light. Equation that is required to compute free space loss $L_{f s}$ can be expressed as,

$$
L_{f s}=20 \log \frac{4 \pi d}{\lambda}
$$

Substituting $c=3 \times 10^{8} \mathrm{~m} / \mathrm{sec}$ into Eq. (3) to obtain the expression for $L_{f s}$ in terms of decibel as [6]. [7],

$$
P_{r}(d B W)=P_{t}(d B W)-L_{f s}(d B)+G_{t}(d B i)+G_{r}(d B i)
$$

where $P_{t}$ is transmitter output power in $d B W$ (decibel watt), $L_{f s}$ is free space loss in decibel $d B, G_{t}$ is transmitting antenna gain in $d B i$ (decibel of isotropic radiator), $G r$ is receive antenna gain in $d B i$ [6],[7]. Molecular constituents in air determines the radio refractive index that is indicated by $n$. Numerically, there is a very small difference compared to unity. Subsequently, to suitably magnify $n$ a modified refractivity index, $N$ is introduced as [8],

$$
N=3.73 \times 10^{5} \frac{e_{v a p}}{T_{0}}+77.6 \frac{P}{T_{0}}
$$

where atmospheric pressure and atmospheric temperature are indicated by $P$ measured in $m p a$ and indicate by T measured in Kelvin respectively. Similarly, $e_{e v p}$ is water vapor pressure (in mbar). The refractivity index decreases linearly as $40 \mathrm{~N}$-unit per kilometer. Radius of curvature of radio wave path at low elevation angle is [8], [9], 


$$
\frac{1}{\rho}=-\frac{d n}{d z}
$$

where $\rho$ and $n$ are radius of earth curvature and refractive index of the atmosphere respectively. Similarly, $d n / d z$ is vertical gradient of refractive index. Effective Earth radius $R_{e}$ can be given as,

$$
\frac{1}{R_{e}}=\frac{1}{k_{f a c} a_{e}}=\frac{1}{a_{e}}+\frac{d n}{d z}
$$

In Eq. (7), $a_{e}=6375 \mathrm{Km}$ is Earth radius, $K_{\text {face }}$ is coefficient of terrestrial radius. Let assume the earth is a flat plane to modify the earth curvature. Subsequently the modified refractivity can be expressed as [1],

$$
M=N+\left(\frac{z}{6375 K m}\right) \times 10^{6}=N+157 z
$$

Using Eq. (8) for gradient of modified refractivity index as,

$$
\frac{\partial M}{\partial z}=\frac{\partial N}{\partial z}+157
$$

The different refractive conditions have been discussed in literature namely sub-refraction, standard refraction, super reflection and trapping. Ray with different refractive gradient conditions ( $N$ gradient and $M$-gradient) are discussed in [5], [10]. The trapping conditions of radio wave propagation creates reflection, refraction and diffraction. The most reported tropospheric ducts are ground based ducts, surface-based duct, elevated duct and evaporation duct as shown in Fig 1 . The lower boundary of $1^{\text {st }}$ two ducts are the surface of the Earth. However, in elevated and evaporation duct, both top and bottom boundaries are upside from the earth surface as discussed in [11]. From the aforementioned trapping duct, the surface-based duct, surface duct and elevated duct occurs for very short time [12].

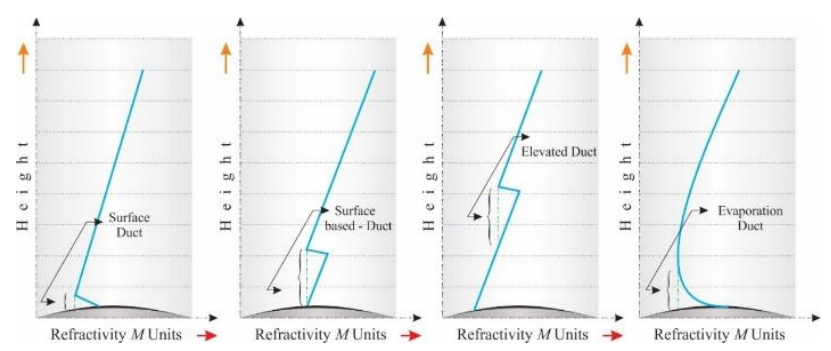

Fig.1. Rays under various refractive conditions

In communication, the irregular terrain surface enables heterogeneity that creates considerable influence on radio wave propagation. Consequentially, the directed EM field will reflect and diffract in a complex way. Because of heterogeneous medium, its need to design an effective communication system that incorporates the refractivity and terrain factors. In order to model radiowave propagation for such complex environment various attempts have been made by researchers. The most challenging task is to model the vast variability of medium electrical properties that allows to re-direct the EM propagation. Previously, Split-step Fourier Transform (SSFM), Finite Element (FE) and Finite Difference methods have been used to predict the radio wave propagation. These methods are computationally inefficient. However, the Split-step Wavelet Transform method (SSWM) is more accurate and computationally efficient [16]. In this research work, the SSWM is proposed for modelling of PWE assuming rough sea surface.

Maxwell's notations are initially assumed that defines the relation of time-varying EM fields travelling through the troposphere. In subsequent section, standard PWE is derived from the Helmholtz's equation.

\section{RADIO wave Propagation}

Basic theory of time varying electromagnetic field is driven from Maxwell's equations. To analyse each component of propagated radio wave, one requires a coordinate system. Subsequently, here we consider spherical coordinate system $(r, \theta, \pi)$ as discussed in [4]. Let consider the earth centre and source is positioned along $z$ axis. Hence Maxwell's equations can be expressed as [13], [15],

$$
\begin{aligned}
& \nabla \times E=j k_{0} Z_{0} H, \\
& Z_{0} . \nabla \times H=-k_{0} j n^{2} E, \\
& \nabla . n^{2} E=0, \\
& \nabla . H=0 .
\end{aligned}
$$

where $n$ is refractivity index of atmosphere, $Z_{o}=120 \pi \Omega$ is impedance of vacuum, $j$ is imaginary constant and $k_{o}=2 \pi / \lambda$ is the wave number, where $\lambda$ is the wavelength. In order to obtain the governing field equation for vertical propagation and horizontal propagation, following assumption are made. Let the EM field components $\left(E_{r}, E_{\theta}, H_{\phi}\right)=0$ for horizontal polarization and resultant equation can be expressed as [15],

$$
\frac{\partial^{2} \psi_{h}}{\partial r^{2}}+\frac{1}{r} \frac{\partial \psi_{h}}{\partial r}+\frac{1}{r^{2}} \frac{\partial^{2} \psi_{h}}{\partial \theta^{2}}+\left(k_{0}{ }^{2} n^{2}-\frac{3}{4 r^{2} \sin ^{2} \theta}\right) \psi_{h}=0
$$

where $\sqrt{k_{0} r \sin E_{\phi}}$ and transforming from spherical plane $(r, \theta)$ to rectangular plane $(x, z)$ as,

$$
\frac{\partial^{2} \psi_{h}}{\partial x^{2}}+\frac{\partial^{2} \psi_{h}}{\partial z^{2}}+k_{0}{ }^{2} n^{2} \psi_{h}(x, z)=0 .
$$

Similarly, the governing field equation for vertical polarization is as [14],

$$
\frac{\partial^{2} \psi_{v}}{\partial r^{2}}+\frac{1}{r} \frac{\partial \psi_{v}}{\partial r}+\frac{1}{r^{2}} \frac{\partial^{2} \psi_{v}}{\partial \theta^{2}}+\left(k_{0}^{2} n^{2}+\kappa\right) \psi_{v}=0,
$$

where in Eq. (16), $\kappa$ can be computed as [15],

$$
\begin{aligned}
\kappa=k_{0}^{2} n^{2} & +\frac{3}{4 r^{2} \sin ^{2} \theta}-\frac{2}{n^{2} r^{2}}\left(\frac{\partial n}{\partial r}\right)^{2} \\
& +\frac{1}{n} \frac{\partial^{2} n}{\partial r^{2}}+\frac{1}{n r^{2}} \frac{\partial^{2} n}{\partial \theta^{2}}-\frac{1}{n r^{2}} \frac{\cos \theta}{\sin \theta} \frac{\partial n}{\partial \theta}-\frac{2}{n^{2}}\left(\frac{\partial n}{\partial \theta}\right)^{2}
\end{aligned}
$$

In Eq. (17), terms $\left[-\left(2 / n^{2}\right)(\partial n / \partial \theta)^{2}+(1 / n)\left(\partial^{2} n / \partial r^{2}\right)\right]$ can be modified as in [15] and expressed as,

$$
-\frac{2}{n^{2}}\left(\frac{\partial n}{\partial \theta}\right)^{2}+\frac{1}{n} \frac{\partial^{2} n}{\partial r^{2}}=\frac{\partial^{2} \log n}{\partial r^{2}}-\left(\frac{\partial \log n}{\partial r}\right)^{2}
$$

Subsequently Eq. (17) can be rewritten as,

$$
\begin{aligned}
\kappa=k_{0}{ }^{2} n^{2}+ & \frac{3}{4 r^{2} \sin ^{2} \theta}+\left(\frac{\partial^{2} \log n}{\partial r^{2}}-\left(\frac{\partial \log n}{\partial r}\right)^{2}\right) \\
& +\left(\frac{1}{n r^{2}} \frac{\partial^{2} n}{\partial \theta^{2}}-\frac{1}{n r^{2}} \frac{\cos \theta}{\sin \theta} \frac{\partial n}{\partial \theta}-\frac{2}{n^{2}}\left(\frac{\partial n}{\partial \theta}\right)^{2}\right)
\end{aligned}
$$

Let assume a stratified atmosphere where $n$ is the function of height then, 


$$
\frac{\partial n}{\partial \theta}=0, \frac{\partial^{2} n}{\partial \theta^{2}}=0
$$

Then Eq. (20) can be modified as,

$\kappa=k_{0}^{2} n^{2}+\frac{3}{4 r^{2} \sin ^{2} \theta}+\left(\frac{\partial^{2} \log n}{\partial r^{2}}-\left(\frac{\partial \log n}{\partial r}\right)^{2}\right)$.

where $\log (n)$ with respect to $r$ is very small so,

$\kappa=k_{0}{ }^{2} n^{2}+\frac{3}{4 r^{2} \sin ^{2} \theta}$

Eq. (16) can be expressed as,

$$
\frac{\partial^{2} \psi_{v}}{\partial r^{2}}+\frac{1}{r} \frac{\partial \psi_{v}}{\partial r}+\frac{1}{r^{2}} \frac{\partial^{2} \psi_{v}}{\partial \theta^{2}}+\left(k_{0}{ }^{2} n^{2}+\frac{3}{4 r^{2} \sin ^{2} \theta}\right) \psi_{v}=0
$$

Transforming Eq. (16) into $(x, z)$ coordinates to obtain expression for vertical polarized field as,

$$
\frac{\partial^{2} \psi_{v}}{\partial x^{2}}+\frac{\partial^{2} \psi_{v}}{\partial z^{2}}+k_{0}^{2} n^{2} \psi_{v}(x, z)=0
$$

where $\psi_{v}=\left(\sqrt{k_{0} r \sin \theta} / n\right) H_{\phi}$ Eq. (15) and (16) are the 2D scalar EM wave equations for propagation of horizontally and vertically polarized field respectively.

\section{Methodology}

Split-Step Wavelet Method (SSWM) and Wavelet Galerkin method have relatively same features excepts for boundary handling [1]. Image theory method based on periodic wavelet function is used to handle the boundary condition in SSWM. Subsequently, geometry of the problem is modified as shown in Fig. 2. Now the range of actual domain with respect to $z$ is changed from $\left[\begin{array}{ll}0 & Z_{\max }\end{array}\right]$ to $\left[\begin{array}{ll}-Z_{\max } & Z_{\max }\end{array}\right]$. The symmetric extension of both field and absorbing layer can be written as,

$$
u\left(x,-Z_{\max }\right)=u\left(x, Z_{\max }\right) \approx 0
$$

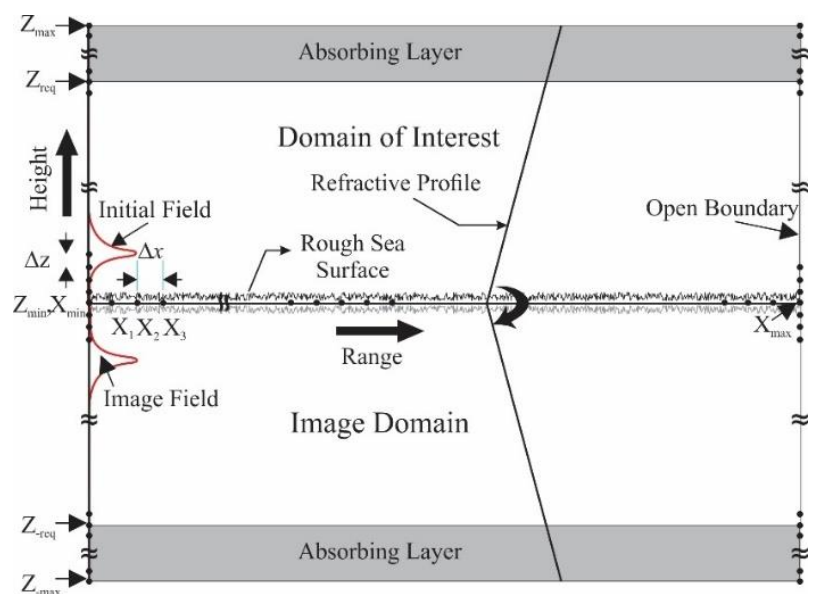

Fig. 2. Extension of solution domain with rough surface using image theory method.

Domain extension satisfies the periodic boundary condition. The periodicity restricts the spatial domain for interval $\left[-Z_{\max } Z_{\max }\right]$ . Now using wavelet expansion of function $u(x, z)$ as,

$$
u(x, z)=\sum_{l=0}^{N_{z}-1} a_{l}(x) \varphi(z-l) ; \quad z \in\left[Z_{-\max }, Z_{\max }\right]
$$

where $a_{l}(x)$ are coefficients which are needs to compute, $\varphi(z)$ are the scaling function. By adopting a procedure that is si(nim)ar to the Wavelet Galerkin Method to obtain equivalent numerical expression as,

$$
\int_{-Z_{\max }}^{Z_{\max }} \varphi_{k}\left(\frac{\partial^{2} u}{\partial z^{2}}+2 j k_{0} \frac{\partial u}{\partial x}+k_{0}^{2}\left(m^{2}-1\right) u\right) d z=0 .
$$

Substitution of Eq. (26) into Eq. (27) as,

$$
\begin{array}{r}
\sum_{l} \frac{\partial a_{l}(x)}{\partial x} \int_{Z_{-\max }}^{Z_{\max }} \varphi_{k} \varphi_{l} d z+\sum_{l} a_{l}(x) \frac{-j}{2 k_{0}} \int_{Z_{-\max }}^{Z_{\max }} \varphi_{k} \frac{\partial^{2} \varphi_{l}}{\partial z^{2}} d z \\
+\sum_{l} a_{l}(x) \frac{-j k_{0}}{2} \int_{Z_{-\max }}^{Z_{\max }}\left(m^{2}-1\right) \varphi_{k} \varphi_{l} d z=0
\end{array}
$$

The matrix notation of Eq. (28) can be expressed as

$$
\left[I_{k, l}\right]\left\{\partial a_{l}(x) / \partial x\right\}+\left[L_{k, l}+S_{k, l}\right]\left\{a_{l}(x)\right\}=0
$$

where,

$$
\begin{aligned}
& I_{k, l}=\delta_{k, l}=\int_{-Z_{\max }}^{Z_{\max }} \varphi_{k} \varphi_{l} d z, \\
& L_{k, l}=\frac{-j}{2 k_{0}} \int_{-Z_{\max }}^{Z_{\max }} \varphi_{k} \frac{\partial^{2} \varphi_{l}}{\partial z^{2}} d z=\frac{-j}{2 k_{0}}\left(\Omega_{l}^{0,2}\right), \\
& S_{k, l}=-\frac{j k_{0}}{2} \int_{-Z_{\max }}^{Z_{\max }}\left(m^{2}-1\right) \varphi_{k} \varphi_{l} d z,
\end{aligned}
$$

In above equations, $\delta_{k, l}$ is the Kronecker delta function and $\Gamma_{l}^{0,2}$ are known as connection coefficient as discussed in [17]. Now using the modified refractivity constant on an element then $S_{k, l}$ can be expressed as [19],

$$
S_{k, l}=-\frac{j k_{0}}{2}\left(m^{2}-1\right) \int_{-Z_{\max }}^{Z_{\max }} \varphi_{k} \varphi_{l} d z
$$

Eq. 27) is an initial value problem. The split-step method derives from the fact that the solution of problem Eq. (27) satisfies the identity

$$
\left\{a_{l}(x+\Delta x)\right\}=\exp (L+S) \Delta x\left\{a_{l}(x)\right\}
$$

The exponential operator given in Eq. (31) can be split in two different ways as,

$$
\left\{a_{l}(x+\Delta x)\right\}=\exp (L \Delta x) \exp (S \Delta x)\left\{a_{l}(x)\right\}
$$

and

$$
\left\{a_{l}(x+\Delta x)\right\}=\exp \left(L \frac{\Delta x}{2}\right) \exp (S \Delta x) \exp \left(L \frac{\Delta x}{2}\right)\left\{a_{l}(x)\right\}
$$

where the form in Eq. (32) in known as asymmetric splitting and the other form given in Eq. (33) in called symmetric splitting. It has been discussed in [20] as asymmetrical splitting is accurate to the order $\mathcal{O}\left(\Delta x^{2}\right)$, where form of symmetric splitting is accurate to the order $\mathcal{O}\left(\Delta x^{3}\right)$.In Eq. (29), $S_{k, l}$ is a diagonal matrix, the exponential of $S_{k, l}$, is required in Eq. (31) that can be solved with less computational time. However, $S_{k, l}$ is a circulant matrix, subsequently its need to compute the exponential of operator $S_{k, l}$. Let,

$$
P=e^{Q} ; Q=L^{\Delta x} / 2
$$

Using the fact that $S_{k, l}$ is circulant, one can compute Eq. (30) by using Fast Fourier Transform (FFT) as discussed in [18],

$$
P={ }^{-1} \exp \left(\Lambda_{q}\right)
$$

where, $\Lambda_{q}=\operatorname{diag}(\hat{q}), \hat{q}=\mathcal{F} q$ and $q$ is first column in Q matrix, and $\mathcal{F}$ is FFT. 


\section{Numerical Implementation of SSWM}

In order to obtain the solution of Eq. (16) with proposed method, the computational domain is assumed to be periodic for an interval of $\left[-Z_{\max } Z_{\max }\right]$. The matrices of Eq. (28) needs to be properly scaled as defined in physical space. Detailed procedure for scaling the solution matrix is provided in [18]. Assuming the solution domain is classified in to $N_{z}$ number of grids points then the indices $l=k=0, \cdots, N_{z}-1$ and the dimensions of resultant obtained linear system of Eq. (28) will be $N_{z} \times N_{z}$. The organization of solution matrix provided in Eq. (28) gives the following structure where length of compactly supported Daubechies wavelet is 6 ,

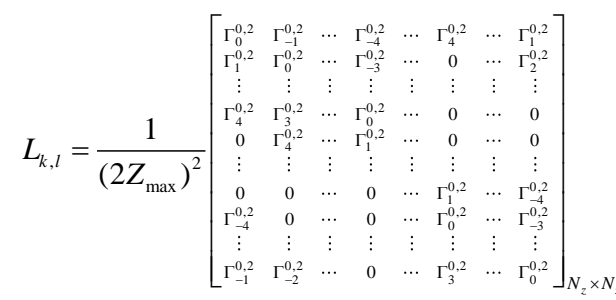

$$
\begin{aligned}
& S_{k, l}=\operatorname{diag}\left[\begin{array}{c}
S_{0,0} \\
\vdots \\
S_{N_{z}-1, N_{z}-1}
\end{array}\right]_{N_{z} \times N_{z}} \text {, } \\
& I_{k, l}=[I]_{N z \times N z} . \\
& \text { and } a_{l}(z)=\left[\begin{array}{lll}
a_{0} & \cdots & a_{N_{z}-1}
\end{array}\right]_{N_{z} \times 1}^{T} \text {. }
\end{aligned}
$$

A symmetric extension of refractive profile is taken in extended domain as shown in Figure 2 and we can write,

$$
S_{\hat{k}, \hat{l}}=S_{k, l} ; k, l=\left(\frac{N_{z}}{2}\right), \ldots, N_{z}-1 ; \hat{k}, \hat{l}=\left(\frac{N_{z}}{2}-1\right), \ldots, 0
$$

Initial field is computed at $x=0$ and in order to satisfy the BCs over perfectly conducting surfaces in SSWM, the unknown coefficients $a_{l}(x)$ are computed for Dirichlet and Neumann BCs as shown in Fig. 2. In order to reduce computational time, the sine and cosine transforms are used for Dirichlet and Neumann BCs, respectively. Since, Split-Step wavelet transform is inspired from Split-step Fourier transform.

Table I: Modified refractive index at certain height from surface

\begin{tabular}{|c|c|c|c|c|c|c|c|c|}
\hline Height (m) & 0.00 & 0.13 & 0.23 & 0.37 & 0.61 & 1.00 & 2.72 & 4.48 \\
\hline M(Units) & 334 & 332 & 331 & 329 & 328 & 327 & 325 & 325 \\
\hline Height (m) & 7.39 & 11.76 & 12.18 & 20.08 & 33.12 & 54.59 & 300.00 & \\
\hline M(Units) & 324 & 324 & 324 & 324 & 324 & 325 & 328 & \\
\hline
\end{tabular}

In order to radiate radio waves through troposphere under evaporation duct phenomenon, the propagation losses are computed at frequency of $3.8 \mathrm{GHz}$ and $7.8 \mathrm{GHz}$ because of prominent trapping conditions in such range. In simulation, rough terrain type is obtained through MATLAB function rand in which the maximum height of the rough terrain is assume $4 \mathrm{~m}$ as illustrated in Fig. 2. The height, polarization and beam-width are assumed $10 \mathrm{~m}$, horizontally and $2^{\circ}$ respectively. Grid sizes along the $X$-axis and $Z$ axis are $200 \mathrm{~m}$ and $0.054 \mathrm{~m}$ respectively. The height verses modified refractive index for evaporation duct is provided in Table 1. The solution for field governing equation is obtained through SSWM and SSFM for comparison. The parameters used in this simulation are summarized in Table II

The propagation of radio waves over variable terrain includes vertically open region. In order to truncate the domain vertically along $Z$-axis, the absorbing boundary conditions are used for yielding non-physical reflection from the top boundary. It is achieved by using window called hamming window. The bottom boundary is assumed to be perfectly electrical conducting earth surface.
Results obtained from proposed method are compared with those from PETOOL under evaporation duct. The PETOOL is a Graphical User Interface developed in MATLAB environment to solve parabolic equation model for one-way and two-way communication. The obtained propagation pattern of radio waves can be seen in Figure 3. It shows the propagation path loss in $\mathrm{dB}$ at frequency of $3.8 \mathrm{GHz}$ computed using SSWM and SSFM. Figure 3(a) and (b) shows the comparison of results from SSWM and SSFM for specified height and range respectively. Similarly, the propagation path loss in $\mathrm{dB}$ at frequency of $7.8 \mathrm{GHz}$ computed using SSWM and SSFM. Figure 3(c) and (d) shows the comparison for specified height and range respectively.

Table II: Simulation Setup for rough surface

\begin{tabular}{|c|c|}
\hline Parameters Value & Value \\
\hline Frequency & $3.8 \mathrm{GHz}$ and $7.8 \mathrm{GHz}$ \\
\hline Range along X-axis & $100 \mathrm{Km}$ \\
\hline Range along z-axis & $300 \mathrm{~m}$ \\
\hline Altitude grid size & $0.054 \mathrm{~m}$ \\
\hline Grid size & $100 \mathrm{~m}$ \\
\hline Beam width & 2 Degrees \\
\hline Antenna Height & $10 \mathrm{~m}$ \\
\hline Polarization Horizontal & Horizontal \\
\hline Elevation angle & 0 Degree \\
\hline Bottom Boundary PEB & PEB \\
\hline Atmosphere parameters & Evaporation Duct \\
\hline
\end{tabular}

In Figure 3(a) and (c), results show a strong agreement with those from SSFM of PETOOL. The strong interference and deep nulls can be seen for the first few kilometers because of strong reflection from variable terrain. The transmission range is extended to several kilometers with very smooth attenuation because of the ducting phenomenon. The percentage of occurrence of evaporation duct is more frequent as compare to another surface-based duct and elevated duct. 

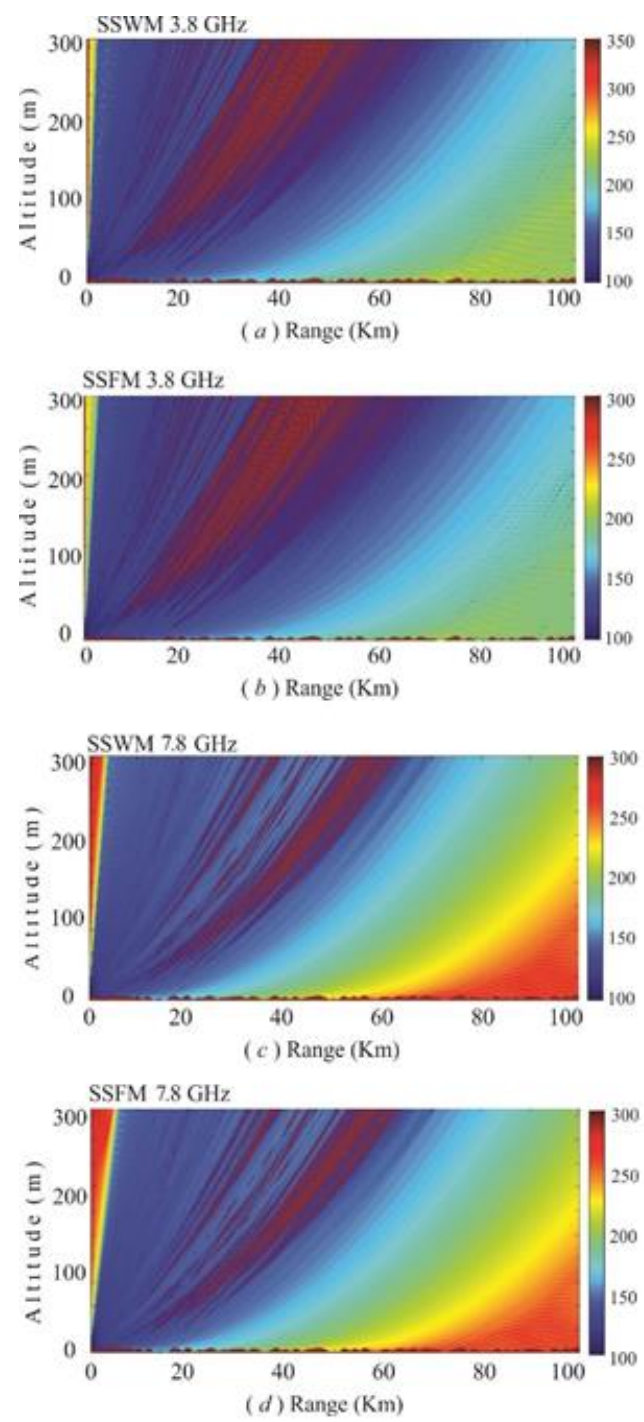

Fig.3: Path loss in $\mathrm{dB}$ for radio wave propagation over the range of 100 $\mathrm{km}$ at $3.8 \mathrm{GHz}$ using (a) SSWM (b) SSFM, at $7.8 \mathrm{GHz}$ (c) SSWM (d) SSFM.

\section{Conclusion}

In this manuscript, SSWM is discussed for modelling of radio wave propagation over rough terrain to estimate path loss in troposphere. The performance of SSWM is demonstrated for standard ducting environment and results are compared with those from PETOOL. It has been concluded that the SSWM allows to estimate reflection and refraction of radio waves because of rough terrain. The obtained results from SSWM for variable terrain shows that the overall pathloss will increase because of roughness, where the increment in loss is proportional to the roughness length The attenuation with selected modelling parameters is increased about $3-4 \mathrm{~dB}$ because of variable terrain. The proposed method also allows to use larger range steps that provides more accurate solution almost as fast as SSFM. In conclusion, the SSWM for radio wave propagation over rough terrain is significant step towards the improvement of propagation model to predict the anomalous propagation in troposphere.

\section{Acknowledgment}

The authors would like to thank the Malaysian Ministry of Higher Education (MOHE) for funding this research work under the Fundamental Research Grant Scheme. The authors also would like thank the Electrical \& Electronic Engineering Department of Uni- versiti Teknologi PETRONAS, Malaysia for their continuous support to this project.

\section{References}

[1] Iqbal, and V. Jeoti. "An Improved Split-Step Wavelet Transform Method for Anomalous Radio Wave Propagation Modelling." Radio Engineering 23(4): 987, 2014

https://www.radioeng.cz/fulltexts/2014/14_04_0987_0996.pdf

[2] Clemmow, P. C. The Plane Wave Spectrum Representation of Electromagnetic Fields: International Series of Monographs in Electromagnetic Waves, Elsevier. 2013, vol. 12

https://courses.engr.illinois.edu/ece458/CPClemmow.pdf

[3] S. Guru, and H. R. Hiziroglu., Electromagnetic field theory fundamentals, Cambridge University Press. 2004, vol. 1

[4] A. Balanis., Antenna theory: analysis and design, John Wiley and Sons. 2015. 4th Edition.

[5] Ghasemi, et al. Propagation Engineering in Wireless Communictions. New York, Springer New York. 2016.

[6] M. Gough., "UHF signal strength measurements as a guide to atmospheric structures." Marconi Review 42: 135-152. 1979

https://www.researchgate.net/publication/238035913_UHF_signal_stren gth measurements_as a guide to atmospheric structures

[7] Lavergnat, and M. Sylvain., Radio wave propagation: principles and techniques, Wiley. 2000

[8] W. Barclay., Propagation of radio waves, Inst of Engineering and Technology. 2012

[9] H. Sizun., Radio wave propagation for telecommunication applications, Springer Verlag.2005

[10] H. R. Reed and C. M. Russell., Ultra high frequency propagation. Quarterly Journal of the Royal Meteorological Society. London,92: 588. 1966

http://onlinelibrary.wiley.com/doi/10.1002/qj.49709239425/abstract

[11] S. Gunashekar., "Transhorizon radio wave propagation due to evaporation ducting." Resonance 11(1): 51-62. 2006

http://www.ias.ac.in/article/fulltext/reso/011/01/0051-0062

[12] T. Manning., Microwave radio transmission design guide, Artech House. 2009

[13] G. Akbarpour., Tropospheric microwave propagation modeling. Department of Electrical and Computer Engineering, The University of Western Ontario (Canada). Ph.D. 2006

[14] O. Ozgun., "PETOOL: MATLAB-based one-way and two-way split-step parabolic equation tool for radio wave propagation over variable terrain." Computer Physics Communications 182(12): 2638-2654. 2011

[15] Mireille. Parabolic equation methods for electromagnetic wave propagation. No. 45. IET, 2000.

[16] B. Hassan, S. Mohammad, and S. Amanallah "A Numerical Study on Optimizing the Geometry and Location of the Openings in Masonry Walls Using Finite Element Method" Journal of Engineering and Applied Sciences 12(9): 2402-2414, 2017

[17] H. Nazabat, et al., "Use of wavelets in marine controlled source electromagnetic method for geophysical modeling." International Journal of Applied Electromagnetics and Mechanics 51.4 : 431-443. 2016

https://content.iospress.com/articles/international-journal-of-appliedelectromagnetics-and-mechanics/jae160002

[18] Ole Møller. Wavelets in scientific computing. Diss. Technical University of Denmark, 1998.

[19] Sirkova and M. Mikhalev., "Parabolic wave equation method applied to the tropospheric ducting propagation problem: a survey." Electromagnetics 26(2): 155-173. 2006

[20] T. Kremp., Split-step wavelet collocation methods for linear and nonlinear optical wave propagation, University of Karlsruhe $(\mathrm{Cu}-$ villier Verlag Göttingen, ISBN 3-89873-605-9). Ph.D. 2002 